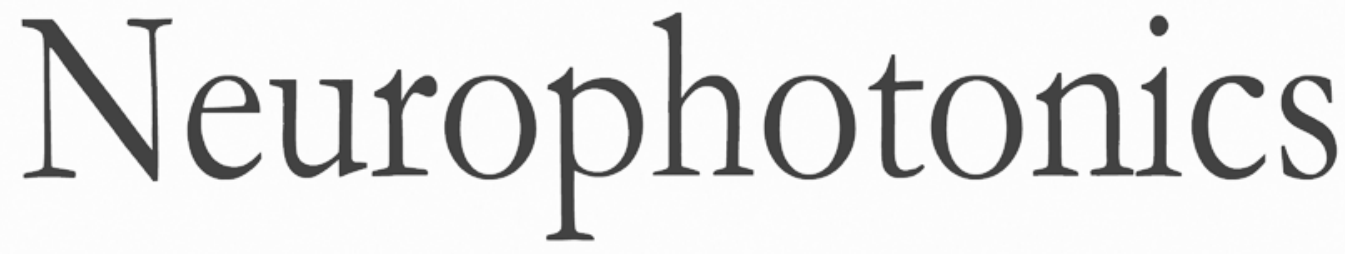

\title{
Intraoperative video-rate hemodynamic response assessment in human cortex using snapshot hyperspectral optical imaging
}

\author{
Julien Pichette \\ Audrey Laurence \\ Leticia Angulo \\ Frederic Lesage \\ Alain Bouthillier \\ Dang Khoa Nguyen \\ Frederic Leblond
}




\title{
Intraoperative video-rate hemodynamic response assessment in human cortex using snapshot hyperspectral optical imaging
}

\author{
Julien Pichette, ${ }^{a, \dagger}$ Audrey Laurence, ${ }^{a, *, \dagger}$ Leticia Angulo, ${ }^{a}$ Frederic Lesage, ${ }^{b}$ Alain Bouthillier, ${ }^{c}$ \\ Dang Khoa Nguyen, ${ }^{d, e}$ and Frederic Leblond ${ }^{a, e}$ \\ aPolytechnique Montreal, Department of Engineering Physics, C.P. 6079, Succ. Centre-Ville, Montréal H3C3A7, Canada \\ ${ }^{b}$ Polytechnique Montreal, Department of Electrical Engineering, C.P. 6079, Succ. Centre-Ville, Montréal H3C3A7, Canada \\ ${ }^{\circ}$ Centre Hospitalier de l'Université de Montréal, Notre-Dame Hospital, Division of Neurosurgery, 1560 Sherbrooke Street East, \\ Montréal H2L4M1, Canada \\ ${ }^{d}$ Centre Hospitalier de l'Université de Montréal, Notre-Dame Hospital, Division of Neurology, 1560 Sherbrooke Street East, \\ Montréal H2L4M1, Canada



\begin{abstract}
Using light, we are able to visualize the hemodynamic behavior of the brain to better understand neurovascular coupling and cerebral metabolism. In vivo optical imaging of tissue using endogenous chromophores necessitates spectroscopic detection to ensure molecular specificity as well as sufficiently high imaging speed and signal-to-noise ratio, to allow dynamic physiological changes to be captured, isolated, and used as surrogate of pathophysiological processes. An optical imaging system is introduced using a 16-bands on-chip hyperspectral camera. Using this system, we show that up to three dyes can be imaged and quantified in a tissue phantom at video-rate through the optics of a surgical microscope. In vivo human patient data are presented demonstrating brain hemodynamic response can be measured intraoperatively with molecular specificity at high speed. ๑ 2016 Society of Photo-Optical Instrumentation Engineers (SPIE) [DOI: 10.1117/1.NPh.3.4.045003]
\end{abstract}

Keywords: hyperspectral imaging; epilepsy; hemodynamic response; intraoperative imaging

Paper 16031R received May 30, 2016; accepted for publication Sep. 19, 2016; published online Oct. 12, 2016.

\section{Introduction}

A variety of optical neuroimaging methods is being developed that may have an important impact on clinical practice. This includes techniques to monitor the functional status of the brain using the intrinsic contrast associated with changes in tissue optical properties following neuronal activity. These hemodynamic changes have been measured using visible and/or near-infrared light, either through-skull, or during open-cranium surgery. ${ }^{1,2}$ The predominant sources of physiologically relevant optical contrast are then associated with the superficial layers of the neocortex. As a result, several groups have been working on the development of methods isolating signal components associated with light-cortex interactions, resulting in optical biomarkers that can be used as surrogates for brain physiological parameters, including changes in blood volume and changes in the oxygen content of blood in the vicinity of active neurons. ${ }^{3-5}$ Depending on the clinical application of interest, the coupling between local cerebral blood flow and oxygen metabolism during neural activation can thus provide an indirect method to measure regional brain neuron activity, which can in some cases be used to inform treatment and/or guide surgical excision of pathological tissue..$^{5-7}$

Here, we present the development and proof-of-concept of an intraoperative optical imaging approach to guide respective surgery for patients with refractory epilepsy. This neurological

*Address all correspondence to: Audrey Laurence, E-mail: audrey.laurence@ polymtl.ca

†The first two authors contributed equally to this work. disorder is characterized by seizures, caused by abnormal excessive neuronal discharges. Patients are candidates for surgical treatment when the area responsible for epileptiform activity is located in a region that can be removed with minimal risk and when seizures cannot be controlled by medication, which happens in about one third of patients. ${ }^{8}$ For those patients, the accuracy of surgical excision is a key factor for postoperative seizure-freedom and improving quality of life while minimizing side effects. Currently, routine preoperative investigations to localize the epileptic focus include magnetic resonance imaging and scalp electroencephalography. During the last diagnostic stage, intraoperative electrocorticography (ECoG) to delineate areas generating interictal epileptiform discharges can also be carried out providing low-resolution $(\sim 1 \mathrm{~cm})$ spatial neuronal activity information for surgical guidance. ${ }^{9}$ Novel intraoperative optical imaging techniques could provide further insights in the neurovascular coupling of this pathology and could potentially complement the information provided by current preoperative imaging and ECoG data to characterize the epileptic focus.

Intrinsic optical imaging techniques rely on wide-field imaging systems with spectroscopic data acquisition capabilities in conjunction with the development of light-transport modeling approaches to isolate the signal contribution of oxygenated hemoglobin ( $\mathrm{HbO}$ ) and deoxygenated hemoglobin (HbR) from light scattering, patient motion as well as physiological phenomena including heart rate and breathing. ${ }^{3,4,10-14}$ Prominent clinical applications of tissue optical spectroscopy include tissue wound healing monitoring ${ }^{15,16}$ and brain hemodynamic

2329-423X/2016/\$25.00 @ 2016 SPIE 

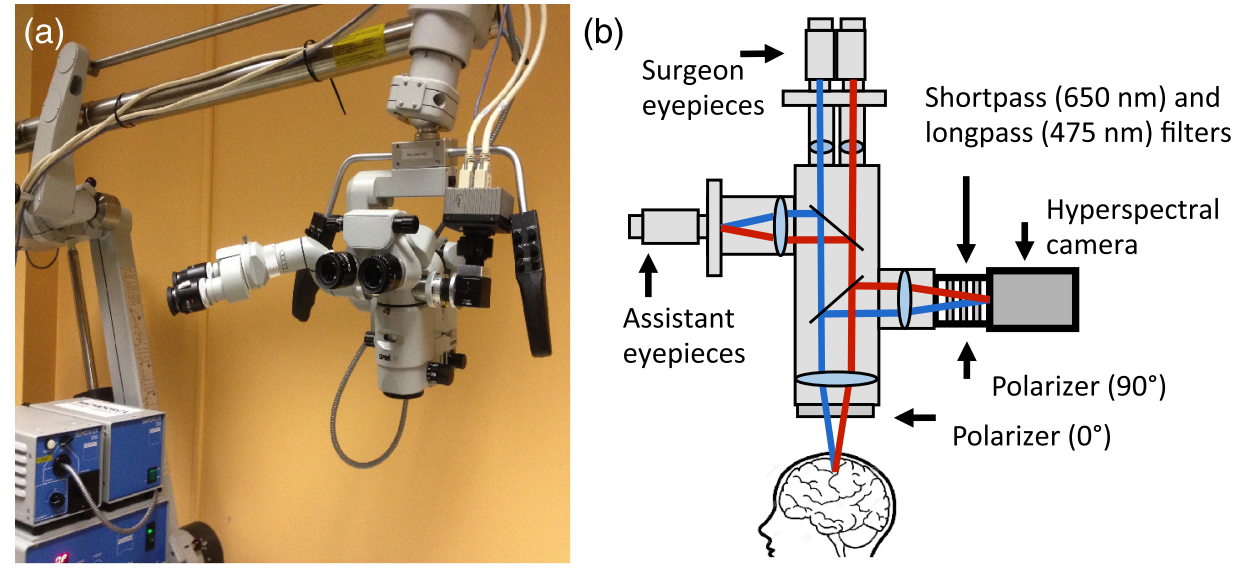

Fig. 1 Neurosurgical imaging system. (a) Hyperspectral camera system connected to a side-port of the neurosurgical microscope. (b) Schematics of the imaging system affixed onto a free optical port.

assessment. $^{12,14,17,18}$ For each of these applications, a range of optical imaging techniques has been developed leading to tissue functional status information, either provided in absolute terms-i.e., by evaluating the concentration of the chromophores-or relative to a baseline value, oftentimes in terms of variations with respect to a signal detected at a specific time point. In brain hemodynamic imaging, the information is either provided topographically with the information effectively being averaged over the penetration depth of the light, ${ }^{3,17,19-24}$ or tomographically exploiting the penetration of visible and/or near-infrared light to provide volumetric information up to a few millimeters or centimeters underneath the surface. ${ }^{14,25-28}$ Most tomographic approaches are implemented through-skull and lead to diffused functional cortex maps, whereas topographic approaches are most often used for intraoperative applications during open-cranium surgery with brain tissue being imaged without the need to correct for light attenuation caused by intervening tissue including skin and skull.

Current intraoperative optical techniques for open-cranium surgical applications are oftentimes associated with limited frame rates and consequently limited signal-to-noise ratios (SNR) due to the limited number of spectral bands that can be acquired simultaneously. ${ }^{3,12,17}$ To circumvent those limitations, here, we present a hyperspectral imaging system using a 16-bands snapshot camera that was developed to monitor intrinsic optical tissue attenuation during neurosurgical procedures. Data acquired on optical phantoms are presented to demonstrate the capabilities of the system to image up to three absorbing dyes simultaneously in real time. Preliminary in vivo human data acquired during a tissue resection procedure are then presented, in which the hemodynamic response to epileptiform activity is measured for a patient with refractory epilepsy.

\section{Methods}

\subsection{Intraoperative Imaging System}

The imaging system is composed of a hyperspectral HSI Snapshot Mosaic sensor (IMEC, Leuven, Belgium) integrating a $4 \times 4$ multispectral filter array (MSFA) disposed over a CMOSIS-CMV2000 CMOS chip. The camera allows simultaneous detection of 16 spectral bands using Fabry-Perot filters with sensitivity ranging from 481 to $632 \mathrm{~nm}$ and a spectral bandwidth (FWHM) of $\sim 15 \mathrm{~nm}$ for each band. The active area of the sensor is composed of $1024 \times 2048$ pixels of $5.5 \mu \mathrm{m}$ across corresponding to $256 \times 512$ "moxels" defined as mosaic elements associated with individual occurrences of the MSFA. The sensor can operate from 10 to 340 frames per second (fps) but for the experiments presented here the camera was operated at either $20 \mathrm{fps}$ (in vivo experiment) or $30 \mathrm{fps}$ (tissue phantom experiments), to be able to acquire images with integration times long enough to ensure sufficient levels of SNR. The effective bit depth of the sensor can be set to 8 or 10 , the full well charge is $13.5 \mathrm{Ke}^{-}$, the dark current is $125 \mathrm{e}^{-} / \mathrm{s}$ (at $25^{\circ} \mathrm{C}$ ), the dark noise is $13 \mathrm{e}^{-}$(RMS) and the fixed pattern noise is $<1$ least significant bit.

For intraoperative use, the hyperspectral camera is connected to a side port of a neurosurgical microscope equipped with a broadband white-light source (Superlux, 300W xenon lamp). Integration of the camera onto the microscope is achieved using a custom optical adapter (TrueTex) (Fig. 1) composed of a polarizer, filters, lenses, and mirrors forming an image of the surgical cavity onto the sensor chip with magnification (1:6 zoom ratio) and focus (working distance ranging from 20 to $50 \mathrm{~cm}$ ) that can be adjusted electronically using a touch-screen display on the microscope. A shortpass filter with cutoff at $650 \mathrm{~nm}$ (Semrock, New York) and a long-pass filter with cutoff at $475 \mathrm{~nm}$ (Edmund Optics, New Jersey) are inserted along the light path within the adapter. Since the sensor's Fabry-Perot filters have leakage in both UV and NIR regions, external cutoff filters are necessary to remove light outside the spectral range of interest. The filters were also selected to reduce the common signal between filters bands, which can decrease the efficiency of the spectral deconvolution algorithms (see Sec. 2.2 for details). Crossed linear polarizers (ThorLabs, New Jersey) are used to minimize the impact of specular reflections. One of the polarizers is installed along the reemission light path, on an adjustable rotation mount within the optical adapter, and the other one is placed in front of the front lens of the neurosurgical microscope directly in-line with the light path of the excitation white-light source. Before every imaging session, the rotational position of the polarizer within the adapter is adjusted to ensure the polarization axes are as close as possible to being perpendicular. Data acquisition with the system is done using the HSImager software (IMEC, Leuven, Belgium) allowing control over camera parameters including integration time and acquisition speed. Data storage and processing are 
achieved using a PC equipped with two $120 \mathrm{~GB}$ solid-state drives mounted in RAID 0 and 16 GB of RAM to allow acquisition speed up to $30 \mathrm{fps}$. All data processing is done using a custom MATLAB (Mathworks) program.

\subsection{Calibration Procedure}

A calibration procedure is implemented on every spatiotemporal and spectral imaging dataset, leading to postprocessed data specific to tissue diffuse reflectance. To compensate for the nonuniform spatial distribution of the light source and to correct for vignetting, every image frame is divided by a measurement made on a Spectralon diffuse reflectance standard (Labsphere, New Hampshire). The standard is placed at the focal plane of the microscope, with the same zoom and focus settings as the main tissue acquisition. A dark noise dataset is acquired with the white-light source turned off. The dark noise is directly subtracted from all imaging datasets prior to normalization by division with the Spectralon dataset.

For each pixel imaged on the surface, the measured spectroscopic data $M_{n}$ in the spectral band $n$ ( $n=1$ to 16 ) can be formally written as a product between the tissue specific diffuse reflectance for that band $S_{n}$ and the instrument response function $\Phi_{n}(\lambda),{ }^{29}$

$M_{n}=\int_{\lambda_{\min }}^{\lambda_{\max }} \Phi_{n}(\lambda) \cdot S_{n} \cdot \mathrm{d} \lambda$,

where the integral runs from the $\lambda_{\min }=473 \mathrm{~nm}$ to $\lambda_{\max }=$ $641 \mathrm{~nm}$, corresponding to the lowest and highest wavelengths for which the system is light sensitive, respectively. The response function can be modeled using the product of the transmission curves of the sensor filters provided by the manufacturer with the spectral response of all other system components including the light source, the bandpass filters, the polarizers, and the optics of the surgical microscope (lenses, dichroic filters, mirrors). Because of second-order responses of the Fabry-Perot filters, there is a significant off-band contribution in the $M_{n}$ measurements [parameterized by the function $\Phi_{n}(\lambda)$ ] that cannot be simply removed by normalization with the Spectralon. Our approach to reduce the impact of those contributions consists of constructing an ideal theoretical instrument response function $\Phi_{n}^{\text {ideal }}(\lambda)$ corresponding to a linear combination of the actual $\Phi_{n}(\lambda)$

$\Phi_{m}^{\text {ideal }}(\lambda)=\sum_{n=1}^{16} x_{m, n} \cdot \Phi_{n}(\lambda)$.

The weighting coefficients $x_{m, n}$ are obtained through mathematical optimization based on the constraint that the ideal response function has its peak located at the same position as $\Phi_{n}(\lambda)$ with a FWHM of $15 \mathrm{~nm}$ but has no off-band contributions. For each ideal band, $m(m=1$ to 16$)$, a least square approach is used to find the coefficients $x_{m, n}$ that minimize the difference between the ideal response and the linear combination in Eq. (2). The resulting $m \times n$ correction matrix

$X=\left(\begin{array}{ccc}x_{1,1} & \cdots & x_{1, n} \\ \vdots & \ddots & \vdots \\ x_{m, 1} & \cdots & x_{m, n}\end{array}\right)$ is then applied to both sides of Eq. (1) leading to a new set of normalized measurements $M_{n}^{\prime}$

$M_{n}^{\prime}=M_{n} X$

representing reconstituted measurements for which the off-band contributions associated with the interference filters have been removed. This provides spectra that are more readily interpretable based on the known basis spectra of tissue chromophores obtained from the literature.

\subsection{Tissue Phantom Experiments}

Experiments were performed using liquid tissue phantoms to validate the calibration procedure outlined in Sec. 2.2 and to develop video-rate spectral deconvolution of multiple absorbing molecules in preparation for in vivo intraoperative hemodynamics imaging experiments (Sec. 2.4). For all phantom experiments, the solutions were prepared using food coloring dyes (blue, red, and yellow, Club House, Canada) and Intralipid ${ }^{\mathrm{TM}}$ (Fresenius Kabi Canada, Canada) mixed in water.

\subsubsection{Experiment 1: basis chromophore spectra and deconvolution technique}

Initially, four solutions were imaged for a single time frame with an integration time of $25 \mathrm{~ms}$. A bulk solution corresponding to Intralipid $^{\mathrm{TM}}$ mixed with water was imaged to be used as a reference measurement. Then, three solutions were prepared and imaged containing either blue, red, or yellow food coloring dyes mixed with Intralipid $^{\mathrm{TM}}$. The absorbers were diluted to obtain solutions with maximum absorption coefficients of $\mu_{a} \cong$ $0.035 \mathrm{~mm}^{-1}$ at 630,526 , or $482 \mathrm{~nm}$ for blue, red, and yellow dyes, respectively. The absorption coefficient of pure dyes was measured using a custom spectrophotometer (CUV-UV Cuvette Holder, Ocean Optics, Maya2000Pro spectrometer, Ocean Optics, white-light LED, ThorLabs). For all solutions, the Intralipid $^{\mathrm{TM}}$ concentration was $0.2 \% \mathrm{~V} / \mathrm{V}$ corresponding to a scattering coefficient of $\mu_{s}^{\prime} \cong 0.23 \mathrm{~mm}^{-1}$ at $630 \mathrm{~nm}$. The calibration procedure was implemented on all four datasets and the absorbance spectra $A(\lambda)$ (a.u.) were obtained using BeerLambert law, with the Intralipid ${ }^{\mathrm{TM}}$-only dataset used as the reference intensity measurement ${ }^{17}$

$A(\lambda)=\log _{10} I_{\text {intra }}(\lambda)-\log _{10} I_{\text {dye }}(\lambda)$,

where labels intra and dye correspond to the Intralipid ${ }^{\mathrm{TM}}$-only and dye + Intralipid $^{\mathrm{TM}}$ measurements, respectively. Normalization with the Intralipid ${ }^{\mathrm{TM}}$-only measurement effectively decouples scattering from absorption insuring $A(\lambda)$ for each dye represents a basis spectrum that can be used for spectral deconvolution following multiplication with the normalization matrix in Eq. (4).

Equal parts of the three solutions (blue, yellow, and red) were mixed together to form a homogenous solution. The solution was imaged and the data normalized as described in Sec. 2.2. Then, a deconvolution technique (least square fit) was applied to each pixel to find the relative contribution of each dye using the basis spectra from Eq. (5). 


\subsubsection{Experiment 2: video-rate spectral deconvolution of multiple absorbers}

Images of a heterogeneous and time-dependent solution (in terms of the concentrations of red, blue, and yellow dyes) were acquired at video-rate to evaluate the capabilities of the instrument to isolate the contribution of multiple chromophores in real time. During image acquisition, dyes in solution were injected from three different syringes into a $30 \mathrm{ml}$ solution of Intralipid $^{\mathrm{TM}}(0.2 \% \mathrm{~V} / \mathrm{V})$. Before the imaging session, each of the syringes was filled with $1 \mathrm{ml}$ of either a blue, red, or yellow solution: $2 \% \mathrm{~V} / \mathrm{V}$ for yellow, $0.2 \% \mathrm{~V} / \mathrm{V}$ for blue and red. Data acquisition was done at $30 \mathrm{fps}$ for $75 \mathrm{~s}$ resulting in an integration time of $25 \mathrm{~ms}$ for each hyperspectral image. The initial injection consisted in $\sim 0.5 \mathrm{ml}$ of the blue dye followed by sequential injection of the same amount of the red and yellow dye solutions. Following this injection sequence, the remaining solutions were injected in an arbitrary manner to ensure all dyes became mixed together forming a complex and highly heterogeneous absorbing medium.

The calibration procedure outlined in Sec. 2.2 was applied to each imaging frame and the absorbance spectra for each pixel was calculated using Eq. (5). As with Experiment 1, spectral deconvolution was applied using the basis spectrum for each dye to extract the relative blue, yellow, and red contribution for every pixel and for each imaged frame. Four $30 \mathrm{fps}$ videos were then produced representing the contribution of each dye as well as reconstituted RGB images to display video information that can be easily interpretable and to illustrate the complexity of the imaged medium. The RGB images were obtained by averaging the signal of different bands to create red (from 582 to $632 \mathrm{~nm}$ ), green (from 526 to $582 \mathrm{~nm}$ ), and blue (from 481 to $526 \mathrm{~nm}$ ) channels.

\subsection{Intraoperative Neurovascular Coupling Assessment}

\subsubsection{Patient selection and intraoperative data acquisition}

The imaging system was used for a 35-year-old female patient undergoing epileptogenic tissue resection at Notre-Dame Hospital (Montreal, Canada) for proof-of-concept demonstration that hemodynamic imaging can be achieved with the intraoperative hyperspectral instrument. The patient received a complete preoperative neurological examination and standard clinical imaging, including ECoG recordings to locate the epileptic focus. Informed consent was obtained from the patient and monitored by the institutional ethics review board. Optical data acquisition was done immediately after craniotomy with the cortex fully exposed, prior to initiating tissue resection. Before the procedure, the camera was connected to the OPMI Pentero surgical microscope (Zeiss, Germany) through an unused optical side port. The data acquisition computer controlling the instrument was located on a cart outside of the sterile area. Spectroscopic imaging recordings were done for $4 \mathrm{~min}$ at a frame rate of $20 \mathrm{fps}$ with an integration time of $40 \mathrm{~ms}$ per frame. Illumination of the surgical field was achieved with the internal microscope white light source set at $100 \%$ intensity. Heart rate and breathing were independently monitored and recorded. ECoG was performed during image acquisition, using electrodes located outside of the field of view of the microscope but near the epileptic focus.

\subsubsection{Calibration procedure and data processing}

The calibration procedure described in Sec. 2.2 (normalization with the Spectralon, data correction with the unmixing matrix $X$ ) was applied to each imaging frame using a custom MATLAB program and a composite RGB image was reconstituted for each frame to display video information reproducing what is seen visually by the surgeon through the microscope eyepieces. All images (for each spectral band) were then corrected for the pulsatile brain motion otherwise causing a relative displacement between the imaging frames and leading to a loss of spatial resolution. An image registration algorithm was implemented using the Medical Image Registration Toolbox for MATLAB. ${ }^{30}$ For each frame, a combination of rigid and nonrigid transformations was calculated to fit the spatial features of a reference frame that was obtained by averaging all frames over time. The spatial deformations were calculated using data at 526 and $592 \mathrm{~nm}$ (average intensity) and the result was applied to all the wavelengths, over a region of interest (ROI) of $235 \times$ 425 pixels. The relative concentrations of $\mathrm{HbO}$ and $\mathrm{HbR}$ were then computed using the calibrated and motion-corrected datasets and a spectral deconvolution technique similar to that presented in Sec. 2.2 was used based on hemoglobin absorption spectra. ${ }^{31}$ However, instead of using the technique in Eq. (5) to account for scattering tissue properties, a pathlength correction was implemented that is based on the method described by Kohl et al. ${ }^{10}$ The method is used to estimate the differential pathlength $\left(D_{a}\right)$ for every imaged pixel, which is a scaling factor to be included in a modified form of the Beer-Lambert law to account for elastic scattering. The measured absorbance variations were modeled

$\Delta A(\lambda)=\sum_{i}\left[\varepsilon_{i}(\lambda) \Delta c_{i} D_{a}(\lambda)\right]$

to take into account the diffusive nature of the tissue and the wavelength dependence of the scattering coefficient. Experimental absorbance changes for each imaging frame are calculated compared to a reference frame, computed by averaging all frames over time. The method consists of five steps: Step 1 consists of modeling the optical properties (absorption and reduced scattering coefficients) of two brain regions labeled "capillaries/cortex" and "blood vessels." Here, "blood vessels" are defined as a region where the optical properties are associated only with hemoglobin, whereas "capillaries/cortex" is defined as a vascularized region composed of brain matter and a small fraction of capillaries. Step 2 consists of using the standard diffusion approximation to radiative transport equation (diffusion equation for a semi-infinite geometry) to calculate the predicted reflectance associated with the optical properties for the two brain regions in step 1. For step 3, images are segmented between "blood vessels" and "capillaries/cortex" regions using the computed reflectance spectra. Step 4 consists of calculating the absorbance spectra associated with the reflectance (in step 2) as a function of tissue optical properties (absorption and reduced scattering coefficients) for each pixel. Step 5 consists of calculating the differential pathlength $\left(D_{a}\right)$ defined as the first derivative of the absorbance with respect to the absorption coefficient. The differential pathlength, which is a function of wavelength, is then used in Eq. (6) to model the absorbance changes due to variations in concentration of $\mathrm{HbO}$ and $\mathrm{HbR}(\Delta \mathrm{HbO}$ and $\Delta \mathrm{HbR})$. 
Absorption coefficients for blood vessels were calculated using a sum of the extinction coefficients of $\mathrm{HbO}$ and $\mathrm{HbR}$ weighted by their relative concentration. ${ }^{31}$ In order to approximate the relative concentration of $\mathrm{HbO}$ and $\mathrm{HbR}$, an oxygen saturation of $99 \%$ was used (read on the oximeter during the surgical procedure) and the baseline value for total hemoglobin concentration used was $150 \mathrm{~g} / \mathrm{L}$. To evaluate the baseline optical properties associated with "capillaries/cortex" regions, we estimated it is composed of $5 \%$ of capillaries with the remainder being associated with brain matter with a baseline absorption coefficient of $0.05 \mathrm{~mm}^{-1}$. $^{32,33}$ The equations for scattering coefficients of both blood (hemoglobin) and brain cortex were $\mu_{s}^{\prime}=$ $22(\lambda / 500)^{-0.66} \mathrm{~mm}^{-1}$ and $\mu_{s}^{\prime}=24.2(\lambda / 500)^{-1.611} \mathrm{~mm}^{-1}$, respectively, with $\lambda$ in nanometer. ${ }^{34}$ Using the reflectance spectra for "blood vessels" and "capillaries/cortex" computed in step 2, a least-square fitting approach was used to compare measured spectra with the reflectance models leading to images segmented between the two region types [Fig. 4(a)]. The images are used to determine which differential pathlength value is to be used for each pixel. More specifically, segmentation was achieved by fitting the theoretical reflectance spectra (calculated with the diffusion approximation equation in step 2) of "blood vessels" and "capillaries/cortex" to each pixel. The differential pathlength for each pixel was then calculated as the sum of the differential pathlength for each region weighed by the proportion of each region type in that pixel.

The cortex exhibits baseline variations in blood flow with temporal frequencies $<\sim 0.2 \mathrm{~Hz}$ (Ref. 35) related with neurovascular coupling such as Mayer waves, vasomotion, and resting state functional connectivity. In order to isolate low frequencies, those associated with breathing and heart rate were removed from the imaging datasets using a low-pass filter $(<0.15 \mathrm{~Hz})$ applied to the time sequences of $\mathrm{HbO}$ and $\mathrm{HbR}$ for each imaged pixel.

\section{Results}

\subsection{Tissue Phantom Experiments}

\subsubsection{Experiment 1: basis chromophore spectra and deconvolution technique}

Figure 2(a) shows the calibrated spectra for the blue, yellow, and red dyes compared with reference absorption spectra of pure dyes measured using a custom hyperspectral spectrophotometer. The calibrated spectra match the reference absorption spectra with a mean-square average error (across the spectral range) of $2.0 \%$ for the blue dye, $2.4 \%$ for the yellow dye, and $1.9 \%$ for the red dye. Maximum discrepancies across the spectral range were $5.7 \%, 4.5 \%$, and $8.2 \%$ for the blue, yellow, and red dyes, respectively.

Figure 2(b) shows data acquired on the homogenous tissue phantom consisting of a solution containing a mixture of all three dyes in equal parts. Calibrated measured data were compared with a fitted spectrum corresponding to a weighed sum of the measured calibrated basis spectra associated with each of the dyes. The weighting coefficients resulting from the fit represent the predicted relative proportion of each dye in the solution, namely 0.25 for the blue dye, 0.20 for the red dye, and 0.22 for the yellow dye. Since the dyes were mixed in equal amounts, the expected coefficients should all have been equal. As a result, the algorithm predicts the correct relative amounts within $\sim 8 \%$.

\subsubsection{Experiment 2: video-rate spectral deconvolution of multiple absorbers}

Spectral unmixing of three dyes (blue, yellow, and red dyes) was then achieved live during video acquisition, as demonstrated in Fig. 3. Images in this figure represent the reconstituted RGB image as well as images in terms of each of the three dyes
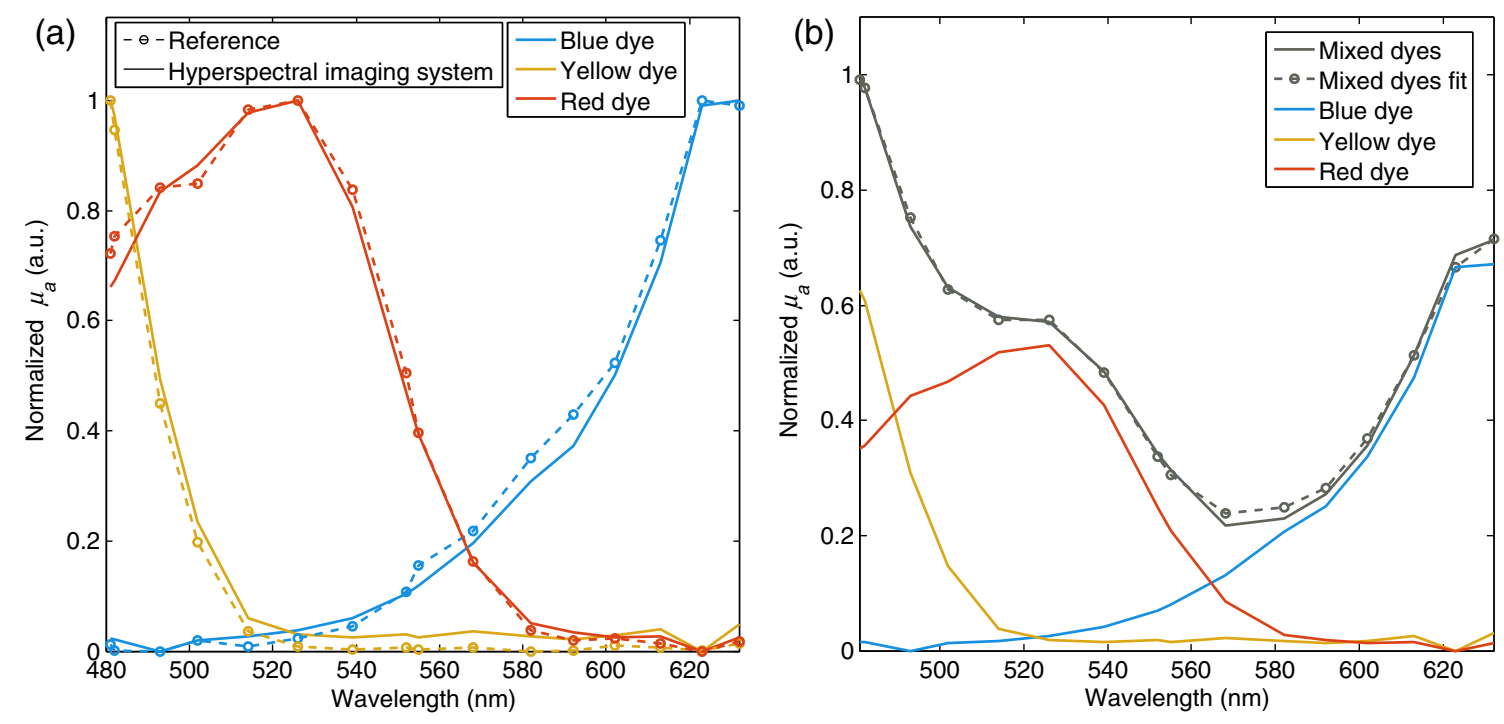

Fig. 2 Tissue phantom experiments. (a) Calibrated spectra (blue, yellow, and red dyes) acquired with the hyperspectral imaging system compared with reference absorption spectra of pure dyes measured using a custom spectrophotometer. (b) Shown in gray are data representing measured (plain line) and fitted (circle markers) spectra of a phantom containing a mixture of the three dyes. The red, yellow, and red curves represent the fitted spectra for each dye with the amplitude representative of their relative concentrations. 


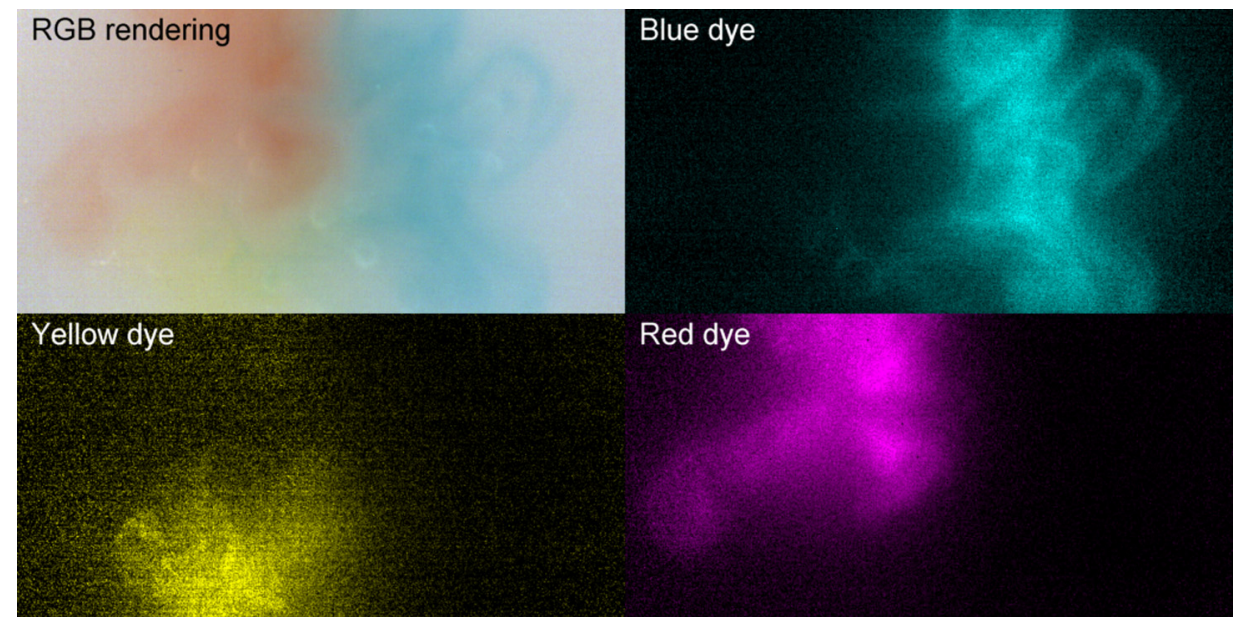

Fig. 3 Spectral unmixing of three dyes (blue, yellow, and red) live during video acquisition. The top-left image is a reconstituted RGB image and the other quadrants show relative dye concentration in cyan, yellow, and magenta (for full video see Video 1, MP4, $15.1 \mathrm{Mb}$ ). Real-time spectral unmixing of three dyes (blue, yellow, and red) (MPEG, 5.9 Mb) [URL: http://dx.doi.org/10.1117/1.NPH.3.4.045003.1].

for only one frame. A full video of the experiment is provided online (Video 1). The observed higher level of noise in the yellow dye image is due to lower gain of the camera within the 480 to $500-\mathrm{nm}$ bands corresponding to the location of the absorption peak of the dye. Figure 3 and Video 1 demonstrate the capabilities of the hyperspectral system to reconstruct at video-rate the relative concentration of different chromophores based on known basis spectra.

\subsection{Intraoperative Neurovascular Coupling Assessment}

Figure 4(b) demonstrates how the differential pathlength correction described in Sec. 2.4.2 can improve the modeling of tissue absorbance changes. Experimental absorbance changes $(\Delta A)$ for one ROI is shown and compared to the reconstituted absorbance after fitting the data with the $\mathrm{HbO}$ and $\mathrm{HbR}$ extinction coefficient spectra with and without the differential pathlength correction. This figure qualitatively shows that the error on the fit is smaller when the differential pathlength correction is applied. Quantitative determination of the improvements was assessed by calculating the average residual between measured data and predicted data across the whole field of view and for the whole duration of the imaging sequence. The residual with the differential pathlength correction is $5.6 \times 10^{-4}$ (a.u.), which is approximately two times smaller than without the pathlength correction $\left[10 \times 10^{-4}\right.$ (a.u.)]. The exclusion criterion that was used for this dataset was that fitted data with standardized residuals (calculated by normalizing the residuals by their standard deviation) $>2$ (absolute value) were excluded, which corresponds to $\sim 5 \%$ of the whole dataset. Figure 5 shows the intraoperative concentration changes of $\mathrm{HbO}, \mathrm{HbR}$, and $\mathrm{HbT}$ for a single time frame. The red regions show an increase in relative concentration, whereas the blue regions show a decrease. Those
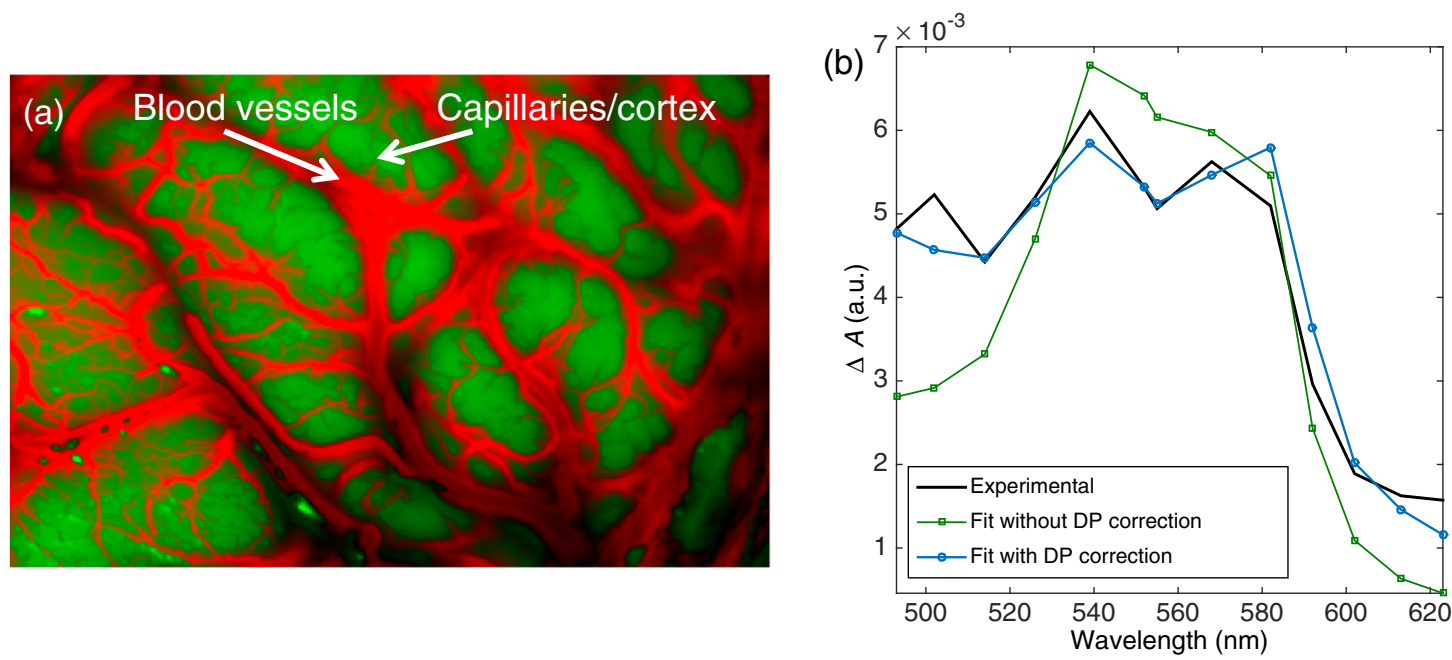

Fig. 4 (a) False-color image of segmented blood vessels (red) and regions with capillaries and cortex. (b) Absorbance changes for one $\mathrm{ROI}$ calculated relative to the average reflectance spectra of this $\mathrm{ROI}$. The $\mathrm{HbO}$ and $\mathrm{HbR}$ absorption spectra were fitted to the experimental data (black line) with (blue-circle markers) and without (green-square markers) differential pathlength (DP) correction. 

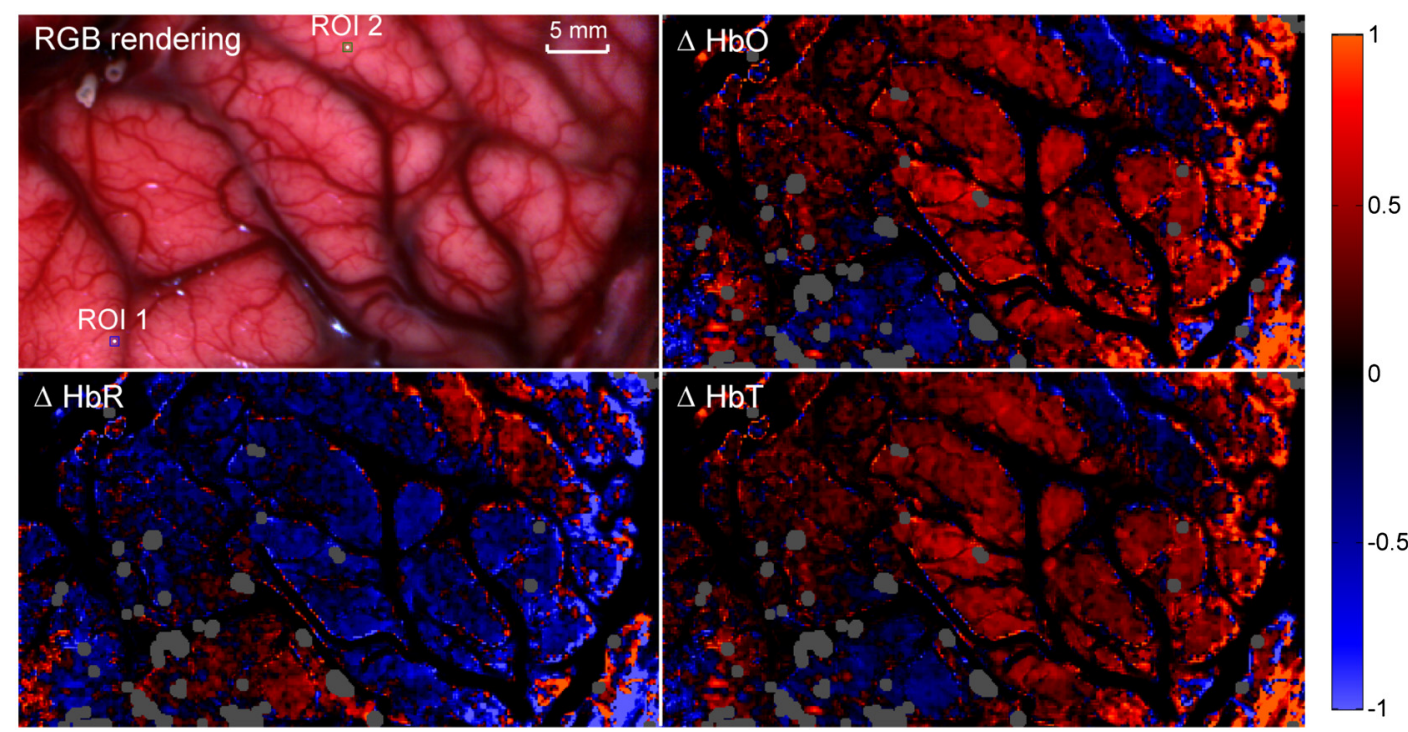

Fig. 5 In vivo relative concentration maps of oxygenated hemoglobin $(\mathrm{HbO})$, deoxygenated hemoblogin $(\mathrm{HbR})$, and total hemoglobin content $(\mathrm{HbT})$ of surface cortex during intraoperative acquisitions. The topleft image is a reconstituted RGB image where two regions of interest are shown (ROI 1 and $R O I 2$ ) where further analysis was made (for full video see Video 2). Real-time in vivo relative concentration maps of oxygenated hemoglobin $(\mathrm{HbO})$, deoxygenated hemoblogin $(\mathrm{HbR})$, and total hemoglobin content $(\mathrm{HbT})$ of surface cortex during intraoperative acquisitions (MPEG, 9.2 Mb) [URL: http://dx.doi.org/10.1117/1.NPH 3.4.045003.2]

changes (decrease or increase) are measured relative to the average frame associated with the whole optical data acquisition sequence. Results are shown for filtered data with only the low-frequency components $(<0.15 \mathrm{~Hz})$. This figure shows that the average regions with an increase in $\mathrm{HbO}$ are associated with regions associated with a decrease in HbR. In order to render more clearly the relatively smaller hemodynamic changes in capillaries, concentration changes in larger blood vessels were artificially attenuated to increase contrast of changes in the cortex. This is represented by dark areas in the $\mathrm{HbO}$, $\mathrm{HbR}$, and $\mathrm{HbT}$ images. In practice, data from the larger blood vessels have stronger (when compared to smaller vessels in the cortex) time-varying components associated with heart rate and breathing, and they are associated with limited levels of SNR due to the high absorption coefficient caused by the large concentrations of hemoglobin. As a result, given the limited dynamic range of the camera used in this work, sufficient SNR allowing robust spectral separation between $\mathrm{HbO}$ and $\mathrm{HbR}$ could be attained only in the cortex. A different acquisition scheme (potentially using a lower frame rate) would be required to ensure sufficient SNR across the brain surface. Areas represented in gray in Fig. 5 correspond to brain regions where camera pixels were saturated due to specular reflections that could not be avoided despite using crossed polarizers in the light paths.

The top-left image in Fig. 5 shows the reconstituted RGB image of the brain surface allowing blood vessels to be visualized. Two regions of interest ( $4 \times 4$ pixels), labeled ROI 1 and ROI 2, were selected to illustrate the frequency response and hemodynamic response of different areas of the brain. The frequency spectrum for those two regions of interest is shown in Fig. 6. Areas of increased spectral power density are highlighted corresponding to: breathing and harmonic component (peaks 1 and 2), and heartbeat and harmonic components (peaks 3, 4, and 5). In order to produce the $\mathrm{HbO}, \mathrm{HbR}$ and $\mathrm{HbT}$ videos in Fig. 5,

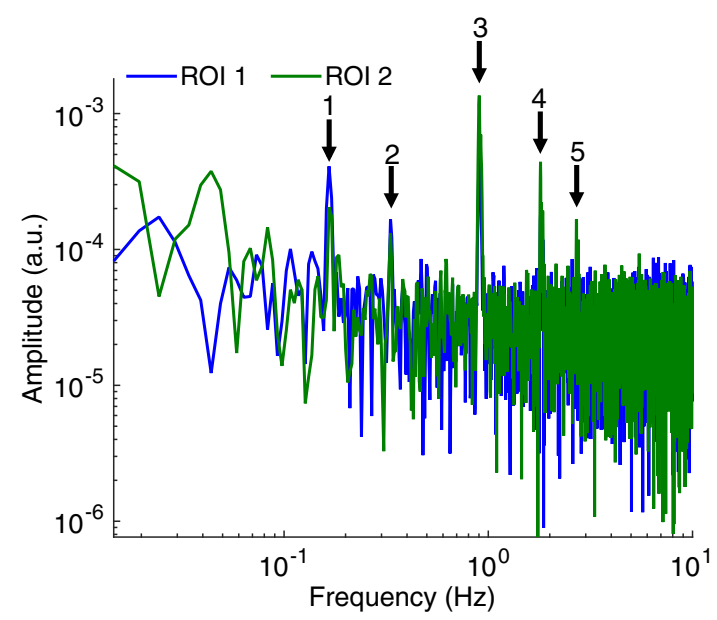

Fig. 6 Frequency spectrum of the hemodynamic response in the two representative regions of interest ( $\mathrm{ROI} 1$ and $\mathrm{ROI} 2)$ shown in Fig. 4. Areas of increased spectral power density are highlighted corresponding to: breathing and one of its harmonic components (peaks 1 and 2), heart rate and harmonic components (peaks 3, 4, and 5).

all frequencies above $0.15 \mathrm{~Hz}$ were removed using a low-pass filter (Fig. 7) resulting in only the very low frequencies being displayed. Figure 8 shows the $<0.15 \mathrm{~Hz}$ time sequences for $\mathrm{HbO}, \mathrm{HbR}$, and $\mathrm{HbT}$ associated with the two regions-of-interest. ECoG recordings indicated the presence of epileptic spikes near the focus during the imaging session. Relative changes in $\mathrm{HbO}$, $\mathrm{HbR}$, and HbT, therefore, possibly represent a combination of Mayer waves, vasomotion, and response to epileptic spikes.

\section{Discussion}

The development of a technique was presented that allows real time hyperspectral imaging in neurosurgery during open-cranium 



Fig. 7 Frequency spectra for ROI 1 (a) and ROI 2 (b) in Fig. 4 with the low frequencies $(<0.15 \mathrm{~Hz})$ represented in darker colors: $\mathrm{HbO}$ (red), $\mathrm{HbR}$ (blue), and $\mathrm{HbT}$ (green). The $>0.15 \mathrm{~Hz}$ power density spectrum is also shown in the background.


Fig. 8 Time-sequences of the hemodynamics associated with the $<0.15 \mathrm{~Hz}$ frequency components for (a) ROI 1 and (b) ROI 2 (see Fig. 4): $\mathrm{HbO}$ (red), HbR (blue), HbT (green).

procedures. The method was demonstrated in tissue-simulating phantoms to quantify the relative concentration of up to three absorbing dyes in a mixture with $<10 \%$ accuracy. Proof-of-concept during an epilepsy surgical resection procedure was then presented demonstrating relative quantification of the variations in oxyhemoglobin $(\mathrm{HbO})$ and deoxyhemoglobin $(\mathrm{HbR})$ in vivo in the human brain. High spatial resolution and high-speed multispectral optical imaging was demonstrated to resolve the time-dependent behavior of blood vessels oxygenation at 20 fps. Beyond the novelty associated with using a snapshot hyperspectral camera technique in neurosurgery for the first time to our knowledge, a new intraoperative data processing method was presented combining: (i) a calibration method specifically adapted to the physical nature of the spectroscopic camera to correct for interband crosstalk, (ii) a registration algorithm to remove brain motion artefacts, (iii) a light transport model-based correction to identify the contributions from reduced scattering and selective absorption, (iv) a spectral unmixing algorithm to quantify the contribution of several chromophores (here $\mathrm{HbO}$ and $\mathrm{HbR}$ ), and (v) a frequency domain filtering operation applied on the time-course of each biomarker to eliminate signal contributions associated with physiological processes such as heart rate and breathing.

Recent progress in brain research led to the realization that baseline variations in blood flow with temporal frequencies between $\sim 0.01$ and $0.15 \mathrm{~Hz}$ are combinations of resting state activity, Mayer waves, vasomotion, and response to other physiological activities. Here, optical data acquired from a single human subject during tissue resection surgery were analyzed. For that patient, direct electrical recordings on the cortex with ECoG were available allowing to locate the epileptic focus and to identify the presence of interictal spikes during image acquisition. Based on this preliminary proof-of-concept work, we can conclude that observed hemodynamic variations are probably a combination of resting-state activities and response to epileptic spikes. The spatial and temporal correlations between those spikes and hemodynamic fluctuations as well as unmixing of the different activities still need to be studied and substantiated based on in-depth analysis in the scope of studies involving larger cohorts of epilepsy patients.

\section{Acknowledgments}

We would like to thank IMEC for technical assistance during the development phase of the instrument. This work was supported by the Discovery Grant program from the Natural Sciences and Engineering Research Council of Canada (NSERC) and the Canadian Foundation for Innovation (CFI).

\section{References}

1. E. M. C. Hillman, "Optical brain imaging in vivo: techniques and applications from animal to man," J. Biomed. Opt. 12(5), 051402 (2007).

2. F. Scholkmann et al., "A review on continuous wave functional nearinfrared spectroscopy and imaging instrumentation and methodology," Neuroimage 85, 6-27 (2014).

3. M. Lavine, M. M. Haglund, and D. W. Hochman, "Dynamic linear model analysis of optical imaging data acquired from the human neocortex," J. Neurosci. Methods 199(2), 346-362 (2011).

4. M. Zhao et al., "Focal increases in perfusion and decreases in hemoglobin oxygenation precede seizure onset in spontaneous human epilepsy," Epilepsia 48(11), 2059-2067 (2007).

5. M. M. Haglund and D. W. Hochman, "Optical imaging of epileptiform activity in human neocortex," Epilepsia 45 Suppl 4(8), 43-47 (2004). 
6. T. Meyer et al., "Intraoperative optical imaging of functional brain areas for improved image-guided surgery," Biomed. Tech. 58(3), 225-236 (2013).

7. T. H. Schwartz, "Neurovascular coupling and epilepsy: hemodynamic markers for localizing and predicting seizure onset," Epilepsy Curr. 7(4), 91-94 (2007).

8. J. W. Miller and S. Hakimian, "Surgical treatment of epilepsy," Continuum (Minneap. Minn). 19(3 Epilepsy), 730-742 (2013).

9. E. Asano et al., "Origin and propagation of epileptic spasms delineated on electrocorticography," Epilepsia 46(7), 1086-1097 (2005).

10. M. Kohl et al., "Physical model for the spectroscopic analysis of cortical intrinsic optical signals," Phys. Med. Biol. 45, 3749 (2000).

11. S. Tak and J. C. Ye, "Statistical analysis of fNIRS data: a comprehensive review," Neuroimage 85 Pt 1, 72-91 (2014).

12. A. Rayshubskiy et al., "Direct, intraoperative observation of $\sim 0.1 \mathrm{~Hz}$ hemodynamic oscillations in awake human cortex: implications for fMRI," Neuroimage 87, 323-331 (2014).

13. M. L. Otten et al., "Motor deficits correlate with resting state motor network connectivity in patients with brain tumours," Brain 135(4), 1017-1026 (2012).

14. A. Sassaroli et al., "Low-frequency spontaneous oscillations of cerebral hemodynamics investigated with near-infrared spectroscopy: a review," IEEE J. Sel. Top. Quantum Electron. 18(4), 1478-1492 (2012).

15. J. T. Nguyen et al., "A novel pilot study using spatial frequency domain imaging to assess oxygenation of perforator flaps during reconstructive breast surgery," Ann. Plast. Surg. 71(3), 308-315 (2013).

16. C. Zhu et al., "Early detection and differentiation of venous and arterial occlusion in skin flaps using visible diffuse reflectance spectroscopy and autofluorescence spectroscopy," Biomed. Opt. Express 7(2), 570-580 (2016).

17. H. J. Noordmans et al., "Imaging the seizure during surgery with a hyperspectral camera," Epilepsia 54(11), e150-e154 (2013).

18. D. Abookasis et al., "Imaging cortical absorption, scattering, and hemodynamic response during ischemic stroke using spatially modulated near-infrared illumination," J. Biomed. Opt. 14(2), 024033 (2009).

19. M. W. Spitzer et al., "Spontaneous and stimulus-evoked intrinsic optical signals in primary auditory cortex of the cat," J. Neurophysiol. 85(3), 1283-1298 (2001).

20. A. K. Dunn et al., "Spatial extent of oxygen metabolism and hemodynamic changes during functional activation of the rat somatosensory cortex," 279-290 (2005).

21. E. Guevara et al., "Optical imaging of acute epileptic networks in mice," J. Biomed. Opt. 18(7), 076021 (2013).
22. H. Ma, M. Zhao, and T. H. Schwartz, "Dynamic neurovascular coupling and uncoupling during ictal onset, propagation, and termination revealed by simultaneous in vivo optical imaging of neural activity and local blood volume," Cereb. Cortex 23(4), 885-899 (2013).

23. M. B. Bouchard et al., "Ultra-fast multispectral optical imaging of cortical oxygenation, blood flow, and intracellular calcium dynamics," Opt. Express 17(18), 15670-15678 (2009).

24. B. R. White et al., "Imaging of functional connectivity in the mouse brain," PLoS One 6(1), e16322 (2011).

25. H. Obrig et al., "spontaneous low frequency oscillations of cerebral hemodynamics and metabolism in human adults," Neuroimage 12(6), 623-639 (2000).

26. T. Näsi et al., "Spontaneous hemodynamic oscillations during human sleep and sleep stage transitions characterized with near-infrared spectroscopy," PLoS One 6(10), e25415 (2011).

27. P. Pouliot et al., "Hemodynamic changes during posterior epilepsies: an EEG-fNIRS study," Epilepsy Res. 108(5), 883-890 (2014).

28. K. Peng et al., "Multichannel continuous electroencephalographyfunctional near-infrared spectroscopy recording of focal seizures and interictal epileptiform discharges in human epilepsy: a review," Neurophotonics 3(3), 031402 (2016).

29. P. Ziemer, "Design and implementation of a multispectral imaging system," PhD Thesis, University of Konstanz (2013).

30. A. Myronenko, "Medical image registration toolbox," (2010), https:// sites.google.com/site/myronenko/research/mirt (11 March 2016).

31. S. A. Prahl, "Tabulated molar extinction coefficient for hemoglobin in water," (1998), http://omlc.org/spectra/hemoglobin/summary.html (11 March 2016).

32. J. D. Johansson, "Spectroscopic method for determination of the absorption coefficient in brain tissue," J. Biomed. Opt. 15(5), 057005 (2010).

33. K. L. Leenders et al., "Cerebral blood flow, blood volume and oxygen utilization," Brain 113(1), 27-47 (1990).

34. S. L. Jacques, "Optical properties of biological tissues: a review," Phys. Med. Biol. 58(11), R37-R61 (2013).

35. M. D. Fox and M. E. Raichle, "Spontaneous fluctuations in brain activity observed with functional magnetic resonance imaging," Nat. Rev. Neurosci. 8(9), 700-711 (2007).

Biographies for the authors are not available. 